\title{
Xanthine Oxidase Inhibits Growth of Plasmodium falciparum in Human Erythrocytes In Vitro
}

Peter A. Berman, Lucille Human, and Janet A. Freese*

Department of Chemical Pathology, University of Cape Town Medical School, Observatory 7925, South Africa; and *Research Institute for Diseases in a Tropical Environment of the South African Medical Research Council, Durban 4001, South Africa

\begin{abstract}
Malaria parasites, unable to synthesize purine de novo, use host-derived hypoxanthine preferentially as purine source. In a previous study (1990. J. Biol. Chem. 265:6562-6568), we noted that xanthine oxidase rapidly and completely depleted hypoxanthine in human erythrocytes, not by crossing the erythrocyte membrane, but rather by creating a concentration gradient which facilitated hypoxanthine efflux. We therefore investigated the ability of xanthine oxidase to inhibit growth of FCR-3, a chloroquine-resistant strain of Plasmodium falciparum in human erythrocytes in vitro. Parasites were cultured in human group $\mathrm{O}^{+}$erythrocytes in medium supplemented, as required, with xanthine oxidase or chloroquine. Parasite viability was assessed by uptake of radiolabeled glycine and adenosine triphosphate-derived purine into protein and nucleic acid, respectively, by nucleic acid accumulation, by L-lactate production, and by microscopic appearance. On average, a $90 \%$ inhibition of growth was observed after $72 \mathrm{~h}$ of incubation in $20 \mathrm{mU} /$ $\mathrm{ml}$ xanthine oxidase. Inhibition was notably greater than that exerted by $10^{-7} \mathrm{M}$ chloroquine $(<\mathbf{1 0 \%}$ ) over a comparable period. The $\mathrm{IC}_{50}$ for xanthine oxidase was estimated at $0.2 \mathrm{mU} /$ ml, compared to $1.5 \times 10^{-7} \mathrm{M}$ for chloroquine. Inhibition was completely reversed by excess hypoxanthine, but was unaffected by oxygen radical scavengers, including superoxide dismutase and catalase. The data confirms that a supply of hostderived hypoxanthine is critical for nucleic acid synthesis in $\boldsymbol{P}$. falciparum, and that depletion of erythrocyte hypoxanthine pools with xanthine oxidase offers a novel approach to treatment of chloroquine-resistant malaria infection in humans. $(J$. Clin. Invest. 1991. 88:1848-1855.) Key words: erythrocyte • hypoxanthine $\bullet$ malaria $\bullet$ oxypurine $\bullet$ Plasmodium falciparum • xanthine oxidase
\end{abstract}

\section{Introduction}

Malaria remains a serious world health problem. An estimated 200 million people suffer from the disease, of whom 1-2 million die annually (1). Effective therapy with antimalarial drugs is hampered by emergence of drug-resistant strains of Plasmodium in Southeast Asia, South America, and, more recently, in Africa (1-4), while immunotherapy is limited by the enormous

Address reprint requests to Dr. Berman, Department of Chemical Pathology, University of Cape Town Medical School, Observatory 7925, South Africa. 1991.

Received for publication 23 April 1991 and in revised form 1 August

J. Clin. Invest.

(C) The American Society for Clinical Investigation, Inc.

$0021-9738 / 91 / 12 / 1848 / 08 \$ 2.00$

Volume 88, December 1991, 1848-1855 diversity of parasite proteins, particularly surface antigens (5). Plasmodium falciparum, a protozoan parasite responsible for the most virulent form of human malaria, lacks the ability to synthesize its purine requirements de novo, and depends on salvage of preformed purines (6). This dependence is shared by many other pathogenic protozoan parasites (7). The relevant pathways of purine metabolism in a malaria parasite and its host erythrocyte are depicted in Figure 1.

Although adenosine triphosphate (ATP) is the predominant purine present in human erythrocytes, there is a considerable body of evidence to indicate that the purine base hypoxanthine, formed from ATP catabolism, is the immediate purine precursor utilized by parasites $(8,9)$. Although the original method for parasite culture described by Trager and Jensen (10), and followed by many workers, does not incorporate additional hypoxanthine, others have shown that supplementation of culture medium with $100-400 \mu \mathrm{M}$ hypoxanthine increases parasite yields three- to fourfold (11-13). Hypoxanthine appeared to be the most important parasite growth promoting component of serum lost after exhaustive dialysis (13). Bungener showed that allopurinol, an inhibitor of xanthine oxidase, marginally enhanced parasite growth in a rat malaria model (14). Radiolabeled hypoxanthine is readily incorporated into nucleic acid by parasites growing in culture $(15,16)$. Enzymes involved in degradation of adenylate nucleotides to hypoxanthine, including adenosine deaminase and purine nucleoside phosphorylase, are produced by $P$. falciparum, which apparently allows it to grow in human erythrocytes deficient in these enzymes $(17,18)$. Enzymes of parasite origin, required for hypoxanthine salvage, including hypoxanthine phosphoribosyltransferase (HPRT), ${ }^{1}$ adenylosuccinate synthase, and inosine monophosphate dehydrogenase, have all been identified (7). Consequently, there appears to exist the complete enzymatic machinery whereby ATP is degraded to hypoxanthine and the hypoxanthine salvaged to form adenylate and guanylate nucleotides. Inhibition of adenosine deaminase by $2^{\prime}$-deoxycoformycin (19), of adenylosuccinate synthase by hadacidin (15), and of inosine monophosphate dehydrogenase by bredinin and mycophenolic acid (15), interfere with parasite growth. This emphasizes the importance of hypoxanthine salvage, as opposed to the more direct salvage of adenosine by adenosine kinase. Adenosine is not a substrate for the plasmodial phosphorylase (18), thus adenosine cannot be salvaged via adenine. Absence of xanthine oxidase in parasite (7) and host cell (20) precludes catabolism of hypoxanthine to urate. Thus, despite some dispute (21), it is now generally accepted that hypoxanthine is an important, if not the exclusive, source of purine for $P$. falciparum. It remains unclear, how-

1. Abbreviations used in this paper: HPRT, hypoxanthine phosphor:bosyltransferase; PRPP, 5-phosphoribosyl 1-pyrophosphate. 


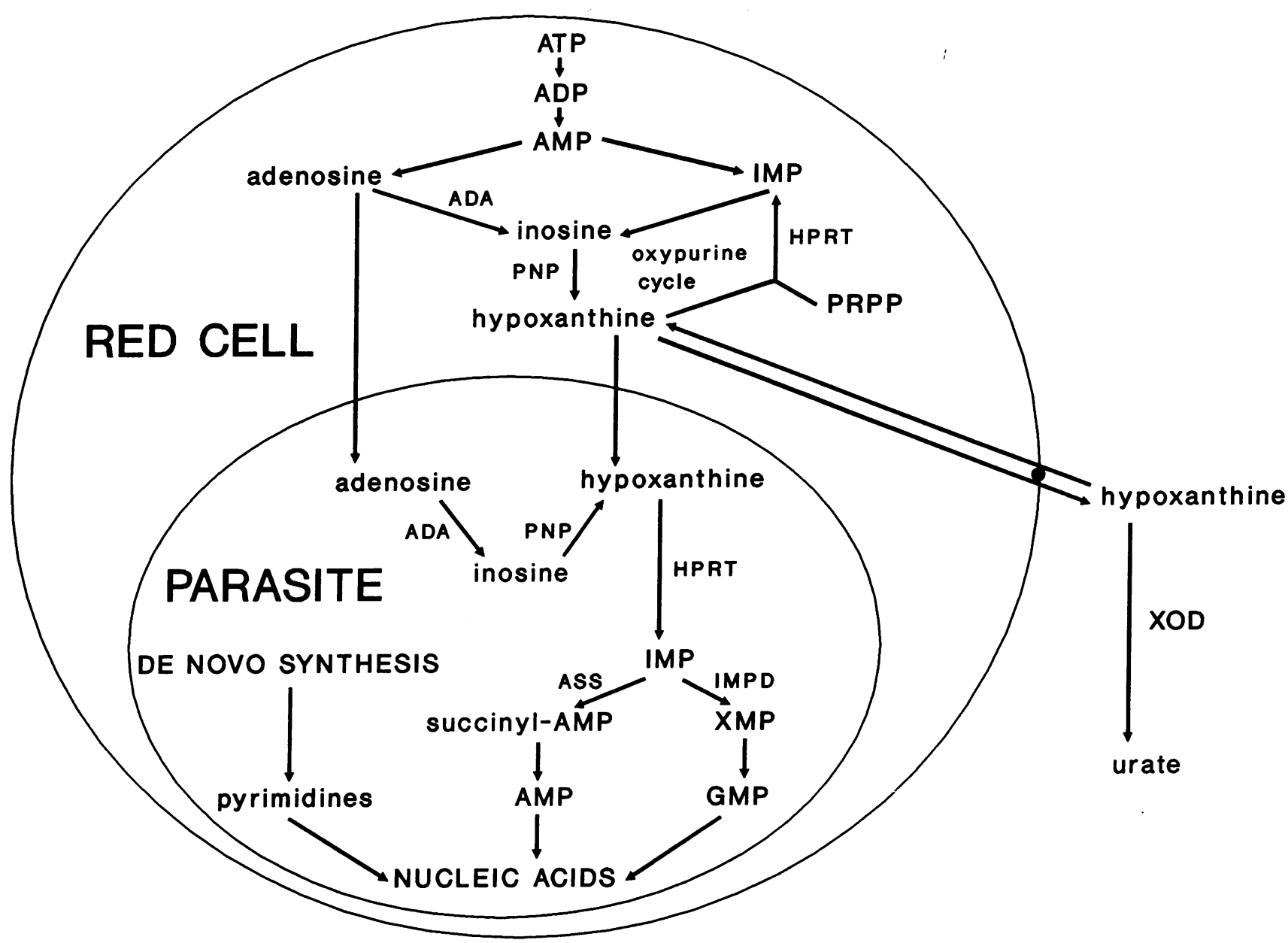

Figure 1. Diagram of a malaria parasite in a human red blood cell (RBC), showing the routes of ATP catabolism in the host and hypoxanthine utilization in the parasite. Hypoxanthine may either enter the parasite to provide the purine moiety of parasite nucleic acid, or diffuse out of the RBC via a high-capacity purine base transporter in the RBC membrane. Efflux of hypoxanthine is facilitated by xanthine oxidase, which oxidizes extracellular hypoxanthine and creates a transmembrane concentration gradient. The nonconcentrative purine base transporter is depicted by the solid circle. Abbreviations: ADA, adenosine deaminase; PNP, purine nucleoside phosphorylase; HPRT, hypoxanthine phosphoribosyl transferase; XOD, xanthine oxidase; PRPP, 5-phosphoribosyl-1-pyrophosphate; ASS, adenylosuccinate synthase; IMPD, inosine monophosphate dehydrogenase.

ever, how the purine needs of the replicating parasite can be supplied by hypoxanthine derived from the host cell, since many studies, including our own, have shown that hypoxanthine release from human red cells under physiologic conditions is too slow to match the rate of parasite nucleic acid synthesis (22-24).

During the course of a previous study on the regulation of hypoxanthine turnover in human erythrocytes, we noted that incubation in xanthine oxidase markedly accelerated the rate of ATP catabolism and hypoxanthine release (22). We suggested that hypoxanthine accumulation and its subsequent release varies inversely with the rate of hypoxanthine recycling through a three-component oxypurine cycle, comprising hypoxanthine, inosine monophosphate, and inosine. Provided that the rate of 5-phosphoribosyl 1-pyrophosphate (PRPP) synthesis, needed for hypoxanthine salvage, matched cycle flux, hypoxanthine accumulation remained negligible. Only when cycle flux exceeded PRPP synthesis, did PRPP levels decline and hypoxanthine efflux begin. Hypoxanthine efflux was tightly coupled to PRPP depletion under a variety of experimental conditions. We proposed that, by irreversibly oxidizing extracellular hypoxanthine, xanthine oxidase established a concentration gradient, which led to the efflux of hypoxanthine via the high-capacity passive purine base transporter located in the red cell membrane. Consistent with this interpretation was the PRPP accumulation provoked by xanthine oxidase, suggesting that the enzyme was able to lower intracellular hypoxanthine to levels insufficient to sustain the HPRT reaction; that is, to levels well below the $K_{\mathrm{m}}$ of $10 \mu \mathrm{M}$ of HPRT for hypoxanthine (25). The same explanation may hold for the findings of Roth et al. (26), who noted a 50-fold increase in PRPP levels of red cells infected with early schizont stages of $P$. falciparum. This stage of parasite development is associated with a sudden burst of nucleic acid synthesis, and also, presumably, of hypoxanthine utilization. Direct measurement in red cells equilibrated with $20 \mu \mathrm{M}\left[{ }^{14} \mathrm{C}\right]$ hypoxanthine confirmed that xanthine oxidase depleted intracellular hypoxanthine to well below $1 \mu \mathrm{M}$ in $<1 \min (22)$.

Since noninfected red cells lack adenylosuccinate synthase, hypoxanthine cannot be reincorporated into adenylate nucleo- 
tides, and is essentially a metabolic end product (9). Hence depletion of hypoxanthine per se would not be expected to exert any deleterious effect on noninfected erythrocytes. Indeed, human tolerance towards very low plasma hypoxanthine seen during renal dialysis suggests that hypoxanthine depletion in general has no serious sequelae in humans.

On the assumption that xanthine oxidase may successfully compete with malaria parasites for available intracellular hypoxanthine, we set out to examine the effect of xanthine oxidase on growth of $P$. falciparum in vitro, and compare it with the widely used antimalarial drug, chloroquine. Chloroquine, a hydrophobic quaternary amine base, diffuses across the red cell and parasite membrane at neutral $\mathrm{pH}$, to become protonated and trapped in the acidic parasite food vacuole (27). Here, probably by increasing the $\mathrm{pH}$ of the vacuole, it interferes with proteolytic digestion of endocytosed red cell cytoplasm, which provides the parasite with the amino acids needed for growth. Chloroquine resistance is based on the expression of an ATPdependent glycoprotein on the parasite membrane that pumps chloroquine out, and maintains a 50-fold lower cytosolic chloroquine concentration than that observed in sensitive strains (3). The broad substrate specificity of this transporter confers resistance to a wide range of antimalarial drugs, often having very different modes of action. We resolved to culture FCR-3, a well-characterized chloroquine resistant strain of $P$. falciparum, and compare the effect of chloroquine and xanthine oxidase on parasite viability, as assessed microscopically, and by a number of independent biochemical parameters. In particular, we were interested in whether growth inhibition caused by xanthine oxidase was due to hypoxanthine depletion or due to formation of active oxygen metabolites, including $\mathrm{O}_{\dot{2}}^{-}$. or $\mathrm{H}_{2} \mathrm{O}_{2}$. In this regard, ability of excess hypoxanthine, and the inability of catalase and superoxide dismutase, to reverse inhibition would confirm the former mechanism.

\section{Methods}

Culture of P. falciparum. FCR-3, a well-characterized chloroquine resistant strain of $P$. falciparum was obtained from the Research Institute for Diseases in a Tropical Environment, Durban, South Africa, and maintained in culture according to the method of Trager and Jensen (10), as modified by Freese et al. (11). Briefly, parasites were grown in $\mathrm{O}^{+}$human erythrocytes, obtained from freshly drawn venous blood after removal of the plasma and buffy coat by repeated washes in medium. Infected erythrocytes were suspended in 20 vol RPMI-1640 medium (Highveld Biological, Kelvin, South Africa), supplemented with Hepes (25 mM), glucose (22 mM), $\mathrm{NaHCO}_{3}(25 \mathrm{mM})$, and human $\mathrm{A}^{+}$ serum $(10 \% \mathrm{vol} / \mathrm{vol})$. Unless specifically indicated, medium was not supplemented with hypoxanthine. Flasks were equilibrated with a gas mixture consisting of $4 \% \mathrm{O}_{2}, 4 \% \mathrm{CO}_{2}, 92 \% \mathrm{~N}_{2}$, and incubated at $37^{\circ} \mathrm{C}$. Medium was replaced daily, and fresh cells were added twice weekly to maintain a parasitemia between $0.1 \%$ and $10 \%$. Percent parasitemia was determined daily on Giemsa-stained (BDH, Poole, UK) thin blood films. All experiments were initiated with unsynchronized cultures at a $1 \%$ parasitaemia.

Incorporation of $\left[{ }^{14} \mathrm{C}\right]$ glycine into parasite protein. Cultures were centrifuged, the medium was discarded, and $0.2-\mathrm{ml}$ aliquots of erythrocytes were resuspended in $1 \mathrm{ml}$ of medium supplemented with $4 \mu \mathrm{M}$ $\left[1-{ }^{14} \mathrm{C}\right]$ glycine (Amersham, Buckinghamshire, UK) $(54 \mathrm{Ci} / \mathrm{mol})$. Glycine was chosen as it is a relatively abundant amino acid in most proteins. Serious error arising from its incorporation into purines and heme were considered unlikely, as neither erythrocytes nor parasites synthesize purines de novo, and mature erythrocytes, which lack mitochondria, are unable to synthesize heme. Suspensions were gassed as described above, and incubated for $5 \mathrm{~h}$. Erythrocytes were lysed in fresh medium containing $0.15 \%$ saponin (Hopkin and Williams, Essex, UK). The red cell ghosts and parasites were deposited by centrifugation, washed twice in fresh medium, then transferred to $2.5-\mathrm{cm}$ diam $\mathrm{GF} / \mathrm{F}$ glass fiber filters (Whatman International, Maidstone, UK), and washed again with $50 \mathrm{ml}$ of distilled water, using a vacuum filtration unit (Millipore Corp., Bedford, MA). The discs were transferred to counting vials containing $5 \mathrm{ml}$ of scintillation fluid (Hionic-Fluor, Packard Instruments Co., Inc., Downers Grove, IL), and counted in a LS 6000IC liquid scintillation counter (Beckman Instruments, Inc., Fullerton, CA).

Incorporation of $\left[{ }^{14} \mathrm{C}\right]$ purine from $\left[{ }^{14} \mathrm{C}\right] \mathrm{ATP}$ into parasite nucleic acid. $5 \mathrm{ml}$ of fresh washed erythrocytes were enriched with $\left[{ }^{14} \mathrm{C}\right] \mathrm{ATP}$ by incubation in $5 \mathrm{ml}$ medium containing $10 \mu \mathrm{M}\left[8-{ }^{14} \mathrm{C}\right]$ adenine (Amersham) $(54 \mathrm{Ci} / \mathrm{mol})$. After $1 \mathrm{~h}, 99 \%$ of the radioactivity had disappeared from the medium, and had been incorporated into the intracellular adenylate nucleotide pool, mainly as ATP, as shown by high pressure liquid chromatography (HPLC) analysis (22). The medium was discarded, and the erythrocytes were infected with $P$. falciparum at a $1 \%$ initial parasitemia. Incorporation of radioactivity into the growing parasites was determined by saponin lysis of erythrocytes and trapping the liberated parasites on filters, as described for $\left[{ }^{14} \mathrm{C}\right]$ glycine.

Conditions for investigating the effect of chloroquine and of xanthine oxidase, with or without hypoxanthine, on nucleic acid and L-lactate accumulation. Six sets of six flasks each were prepared, all containing $0.2 \mathrm{ml}$ of freshly drawn, washed erythrocytes, and $4 \mathrm{ml}$ of medium. Sets 2 and 3 were supplemented with chloroquine to give a final concentration of $10^{-7}$ and $5 \times 10^{-7} \mathrm{M}$, respectively. Sets 4 and 5 were supplemented with $3 \mathrm{mU} / \mathrm{ml}$ xanthine oxidase, while set 5 contained, in addition, $1 \mathrm{mM}$ hypoxanthine. Sets $1-5$ were inoculated with $20 \mu \mathrm{l}$ of infected erythrocytes ( 10\% parasitemia). Set 6 served as noninfected control. All flasks were gassed and incubated at $37^{\circ} \mathrm{C}$. Every $24 \mathrm{~h}$, the red cells were centrifuged and the medium removed and kept for assay of L-lactate. Two members of each set were processed for nucleic acid content, while the remainder were resuspended in fresh medium of the appropriate composition, gassed, and reincubated.

Nucleic acid extraction and quantitation. The $0.2 \mathrm{ml}$ of cell pellets was lysed in $6 \mathrm{ml}$ of a cell lysis buffer, consisting of Tris-HCl $10 \mathrm{mM}$, pH 7.6, sucrose $0.32 \mathrm{M}, \mathrm{MgCl}_{2} 5 \mathrm{mM}$, and Triton X-100 $1 \%$ (vol/vol). The lysate was centrifuged at $2,500 \mathrm{~g}$ for $20 \mathrm{~min}$, and the insoluble pellet, consisting mainly of parasites and degraded heme, was washed once in $2 \mathrm{ml}$ of the same buffer. The pellet was resuspended in $0.6 \mathrm{ml}$ of a digestion buffer containing EDTA $25 \mathrm{mM}, \mathrm{pH} \mathrm{8.0,} \mathrm{NaCl} 75 \mathrm{mM}$, SDS $1 \%(\mathrm{wt} / \mathrm{vol}$ ), and Proteinase $\mathrm{K}$ (Boehringer Mannheim, GmbH, FRG) $0.5 \mathrm{mg} / \mathrm{ml}$, and incubated overnight at $56^{\circ}$ to ensure complete proteolysis. The suspension was mixed with $0.15 \mathrm{ml}$ of saturated sodium acetate to "salt out" degraded protein, and centrifuged at 10,000 $g$ for $10 \mathrm{~min}$ at $10^{\circ} \mathrm{C}$ to obtain a clear supernatant containing nucleic acid. The supernatant was mixed well with an equal volume of isopropanol, and kept at $-20^{\circ} \mathrm{C}$ for $2 \mathrm{~h}$ to allow complete precipitation of nucleic acid. The nucleic acid pellet was washed once with cold $70 \%$ ethanol, and dissolved in $1 \mathrm{ml}$ of distilled water. The ultraviolet (UV) absorption spectrum of the extract was measured, and the nucleic acid concentration was calculated from the absorbance at $260 \mathrm{~nm}$, assuming that a $50 \mu \mathrm{g} / \mathrm{ml}$ solution of nucleic acid has an absorbance of $1.0 \mathrm{U}$ at this wavelength (28).

Agarose gel electrophoresis. $20 \mu \mathrm{l}$ of nucleic acid extract (up to $1 \mu \mathrm{g}$ nucleic acid) was electrophoresed for $16 \mathrm{~h}$ at $40 \mathrm{~V}$ on a $2 \%$ agarose gel (Sigma Chemical Co., St. Louis, MO). Tris acetate $40 \mathrm{mM}, \mathrm{pH} \mathrm{8.0,}$ containing $2 \mathrm{mM}$ EDTA, was used to prepare the agarose and provide tank buffer. The gel was stained for $1 / 2 \mathrm{~h}$ in $10 \mu \mathrm{g} / \mathrm{ml}$ ethidium bromide, destained in water for $1 / 2 \mathrm{~h}$, visualized under UV light, and photographed.

Supplementation of medium. Where indicated, medium was supplemented with xanthine oxidase (Boehringer) at a concentration of 20 $\mathrm{mU} / \mathrm{ml}(0.1 \mathrm{mg} / \mathrm{ml})$, except in the hypoxanthine rescue experiment, where its concentration was reduced to $3 \mathrm{mU} / \mathrm{ml}$. Superoxide dismutase and catalase (Sigma Chemical Co.) was used at concentrations of 
0.4 and $0.15 \mathrm{mg} / \mathrm{ml}$, respectively. Chloroquine sulfate (Sigma Chemical Co.) was diluted from a $1 \mathrm{mM}$ aqueous stock solution to give the appropriate final concentration.

Measurement of medium L-lactate concentration. L-Lactate was measured on perchloric acid extracts of medium, by monitoring the formation of NADH in the presence of lactate dehydrogenase (29).

Procedure for determining the extent of hypoxanthine oxidation under hypoxanthine "rescue" conditions. Culture medium containing $3 \mathrm{mU} / \mathrm{ml}$ xanthine oxidase and $25 \mu \mathrm{M}\left[{ }^{14} \mathrm{C}\right]$ hypoxanthine $(54 \mathrm{Ci} / \mathrm{mol})$ was incubated at $37^{\circ} \mathrm{C}$ for $1 \mathrm{~h}$ in the absence, or $24 \mathrm{~h}$ in the presence, of $1 \mathrm{mM}$ unlabeled hypoxanthine. Medium was deproteinized with 0.25 vol $1.5 \mathrm{M}$ perchloric acid, and the clear supernatant was neutralized with $6 \%$ (vol/vol) $2.5 \mathrm{M} \mathrm{K}_{2} \mathrm{CO}_{3}$. After allowing the $\mathrm{KClO}_{4}$ precipitate to settle overnight at $4^{\circ} \mathrm{C}$, the distribution of label in the supernatant was analysed by reverse-phase HPLC.

HPLC. Separation of oxypurine metabolites was effected by HPLC of neutralized perchloric acid extracts of medium, using a model SF 8800 ternary HPLC pump (Spectra Physics, Inc. Mountain View, CA) and a C-18 Novapak $4 \mathrm{u}$ reverse-phase cartridge, mounted in a radial compression module (Waters Associates, Inc., Milford, MA). Elution was performed isocratically with $0.15 \mathrm{M} \mathrm{NH}_{4} \mathrm{H}_{2} \mathrm{PO}_{4}, \mathrm{pH} \mathrm{6.0,} \mathrm{at} \mathrm{a} \mathrm{flow}$ rate of $1.5 \mathrm{ml} / \mathrm{min}$. Retention times of urate, hypoxanthine, and xanthine were determined with authentic standards, by monitoring absorbance of the effluent at $260 \mathrm{~nm}$ (UV-vis detector, model SP 8450 , Spectra Physics). After injection of ${ }^{14} \mathrm{C}$-labeled samples, 0.25 -ml fractions of the eluate corresponding to the purines of interest were collected, mixed with scintillant, and counted as described under Incorporation of $\left[{ }^{14} \mathrm{C}\right]$ glycine.

\section{Results}

Effect of xanthine oxidase on $\left[{ }^{14} \mathrm{C}\right]$ glycine incorporation into protein. Parasites grown in the absence of xanthine oxidase showed a progressive increase in their ability to incorporate $\left[{ }^{14} \mathrm{C}\right]$ glycine into protein (Fig. $2 \mathrm{~A}$ ). Incorporation was markedly impaired by the presence of xanthine oxidase, so that by the third day, protein synthesis had decreased to the negligible levels observed in noninfected cells. At the conclusion of the experiment, mean parasitemia, measured on Giemsa-stained films, was $5.0 \%$ and $0.3 \%$, for infected cells incubated in the absence and presence of xanthine oxidase respectively.

Effect of xanthine oxidase on purine incorporation from $\left[{ }^{14} \mathrm{C}\right]$ ATP into nucleic acid. Parasites were grown in red cells in which the adenylate nucleotide pool had been prelabeled with $\left[{ }^{14} \mathrm{C}\right]$ adenine. The extent of $\left[{ }^{14} \mathrm{C}\right]$ purine incorporation into nucleic acid increased steadily in parasites incubated in medium alone, whereas incorporation was markedly impaired in parasites exposed to xanthine oxidase. The background level of incorporation of $\left[{ }^{14} \mathrm{C}\right] \mathrm{ATP}$-derived purine into noninfected red cells remained low throughout (Fig. $2 B$ ).

Effect of heat denaturation and oxygen radical scavengers on inhibition by xanthine oxidase. Xanthine oxidase, exposed to $100^{\circ} \mathrm{C}$ for $10 \mathrm{~min}$, lost both enzyme activity and the ability to inhibit $\left[{ }^{14} \mathrm{C}\right]$ glycine incorporation (results not shown). In contrast, superoxide dismutase and catalase had no effect on the inhibition of $\left[{ }^{14} \mathrm{C}\right]$ glycine incorporation exerted by xanthine oxidase (results not shown).

Effect of hypoxanthine on parasites exposed to xanthine oxidase. Fig. 3 shows total nucleic acid content of erythrocytes incubated for $3 \mathrm{~d}$ under a variety of conditions. A steady increase in nucleic acid was observed in infected cells incubated in medium alone. Inclusion of $1 \times 10^{-7} \mathrm{M}$ chloroquine had little effect, whereas $5 \times 10^{-7} \mathrm{M}$ chloroquine markedly inhib-

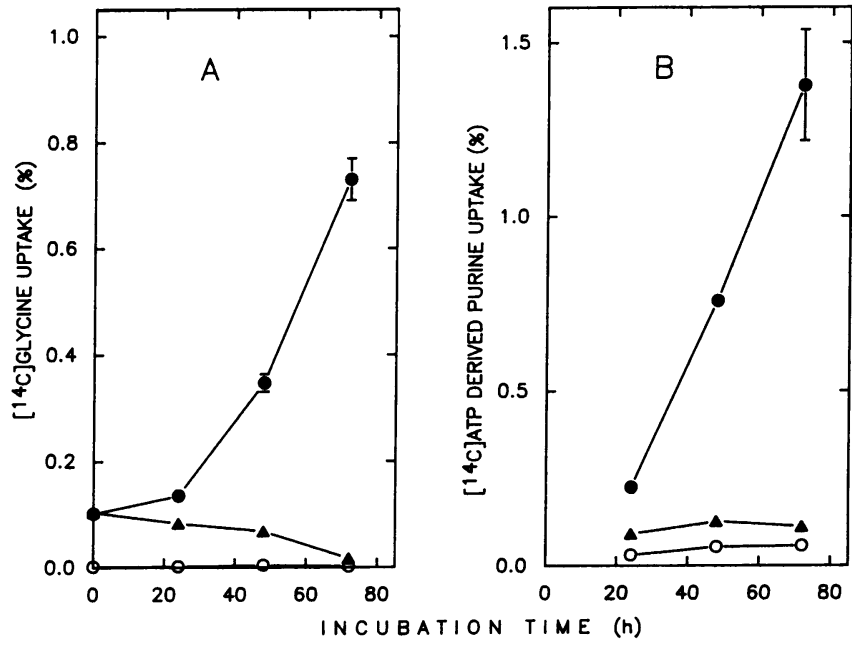

Figure 2. Inhibition by xanthine oxidase of parasite protein and nucleic acid synthesis. $(A)$ Time course of $\left[{ }^{14} \mathrm{C}\right]$ glycine incorporation. Infected RBC, at a $1 \%$ initial parasitemia, were incubated for $72 \mathrm{~h}$, either in the absence ( $\bullet$ ) or presence $(\Delta)$ of $20 \mathrm{mU} / \mathrm{ml}$ xanthine oxidase. Noninfected RBC, incubated without xanthine oxidase, were included as a negative control (o). At timed intervals, aliquots of RBC were resuspended in medium containing $\left[{ }^{14} \mathrm{C}\right]$ glycine, and the percent incorporation of $\left[{ }^{14} \mathrm{C}\right] \mathrm{glycine}$ into parasite protein measured as described in Methods. Each assay was performed in duplicate. Error bars show the values of individual duplicates. Absence of error bars indicate duplicates that fell within the symbol used to depict the mean value. $(B)$ Time course of $\left[{ }^{14} C\right]$ purine incorporation by parasites growing in $R B C$ containing $\left[{ }^{14} \mathrm{C}\right] \mathrm{ATP}$. Fresh, noninfected $R B C$ were prelabeled with $\left[{ }^{14} \mathrm{C}\right] \mathrm{ATP}$ by preincubation with $\left[{ }^{14} \mathrm{C}\right]$ adenine, then infected with $P$. falciparum to give a $1 \%$ initial parasitemia. Incubation was continued for $72 \mathrm{~h}$, either in the absence $(\bullet)$ or presence $(\Delta)$ of $20 \mathrm{mU} / \mathrm{ml}$ xanthine oxidase. Aliquots were removed at timed intervals, and the $\left[{ }^{14} \mathrm{C}\right]$ purine content of parasite nucleic acid measured as described in Methods. Noninfected RBC (0) were incubated in parallel. Results are expressed as a percentage of total RBC $\left[{ }^{14} \mathrm{C}\right] \mathrm{ATP}$ content. Each point is the mean of duplicate estimates, with individual values denoted by error bars.

ited nucleic acid synthesis. Xanthine oxidase, used here at a concentration of $3 \mathrm{mU} / \mathrm{ml}$, exerted a similar inhibitory effect, while inclusion of $1 \mathrm{mM}$ hypoxanthine with $3 \mathrm{mU} / \mathrm{ml}$ xanthine oxidase restored nucleic acid synthesis to levels exceeding those observed in infected cells incubated in medium alone. These results are confirmed by agarose gel electrophoresis of the nucleic acid extract (Fig. 4). A band of high molecular weight DNA is apparent in extracts from control and $100 \mathrm{nM}$ chloroquine-treated cultures, and increases in intensity with time. DNA accumulation is clearly inhibited in extracts from cultures treated with $500 \mathrm{nM}$ chloroquine or with xanthine oxidase, whereas the DNA band is restored to its former intensity in cultures treated with xanthine oxidase plus hypoxanthine. The same pattern emerged when we examined lactate release into the medium over the three 24-h incubation periods (Fig. 5).

Extent of hypoxanthine oxidation under hypoxanthine "rescue" conditions. In order to show that, although xanthine oxidase could effectively oxidize micromolar concentrations of hypoxanthine, it was unable to quantitatively remove the 1 $\mathrm{mM}$ hypoxanthine added to rescue parasites, medium containing $3 \mathrm{mU} / \mathrm{ml}$ xanthine oxidase was incubated with $25 \mu \mathrm{M}$ 


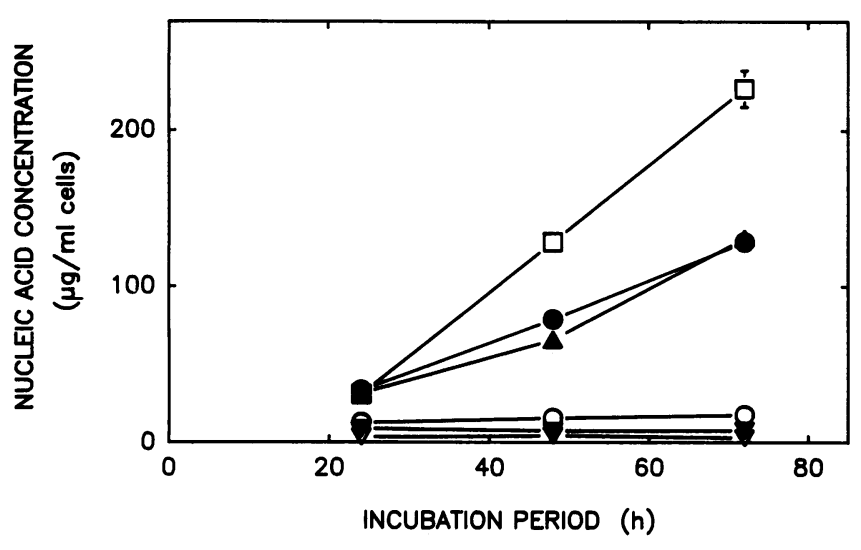

Figure 3. Accumulation of nucleic acid in infected RBC, inhibition by high-dose chloroquine and xanthine oxidase, reversal by hypoxanthine. Infected RBC were incubated in medium alone ( $\bullet)$, in $100 \mathrm{nM}$ chloroquine ( $\triangle$ ), in $500 \mathrm{nM}$ chloroquine $(\nabla)$, in $3 \mathrm{mU} / \mathrm{ml}$ xanthine oxidase (o), or in $3 \mathrm{mU} / \mathrm{ml}$ xanthine oxidase plus $1 \mathrm{mM}$ hypoxanthine ( $\square$ ). Noninfected RBC were included as a control ( $\nabla)$. RBC nucleic content was measured at times indicated. Differences between duplicate analyses, when larger than the symbol used to denote their mean, are shown by error bars.

$\left[{ }^{14} \mathrm{C}\right]$ hypoxanthine for $1 \mathrm{~h}$ (Fig. $6 \mathrm{~A}$ ), or with $1 \mathrm{mM}\left[{ }^{14} \mathrm{C}\right]-$ hypoxanthine for $24 \mathrm{~h}$ (Fig. $6 \mathrm{~B}$ ). HPLC analysis, showing the distribution of radioactivity among hypoxanthine, xanthine, and urate, confirmed that, while $25 \mu \mathrm{M}$ hypoxanthine was completely oxidized to urate within $1 \mathrm{~h}, 16 \%$ of $1 \mathrm{mM}$ hypoxanthine, or $160 \mu \mathrm{M}$, remained present after $24 \mathrm{~h}$ of incubation.

Microscopic appearance of parasites exposed to xanthine oxidase. Giemsa stained blood films were examined, and showed that while ring forms of the parasite predominated in control and chloroquine-treated cultures, xanthine oxidase caused parasites to accumulate as late trophozoites and as schizonts with only two nuclei. The normal distribution of parasite stages, with ring forms predominating, was restored by hypoxanthine rescue.

Dose-response curves for xanthine oxidase and chloroquine. The efficacy of xanthine oxidase and chloroquine as inhibitors of parasite growth was assessed by measuring incorporation of $\left[{ }^{14} \mathrm{C}\right]$ glycine into parasites exposed for $48 \mathrm{~h}$ to varying concentrations of either agent (Fig. 7). Results are expressed as a percentage of the $\left[{ }^{14} \mathrm{C}\right]$ glycine incorporated into parasites incubated in medium alone. The $\mathrm{EC}_{50}$ for xanthine oxidase and chloroquine was estimated at $0.3 \mathrm{mU} / \mathrm{ml}(1.5 \mu \mathrm{g} / \mathrm{ml})$ and 1.5 $\times 10^{-7} \mathrm{M}$, respectively.

\section{Discussion}

We have shown, by a number of independent biochemical approaches, that xanthine oxidase inhibits growth of $P$. falciparum under in vitro culture conditions. Thus, the ability to synthesize protein, the ability to utilize ATP for nucleic acid biosynthesis, overall metabolic activity as indicated by lactate production, and organism number, as reflected by nucleic acid content, are all significantly decreased when infected erythrocytes are exposed to xanthine oxidase. These experiments are greatly facilitated by the fact that mature human erythrocytes

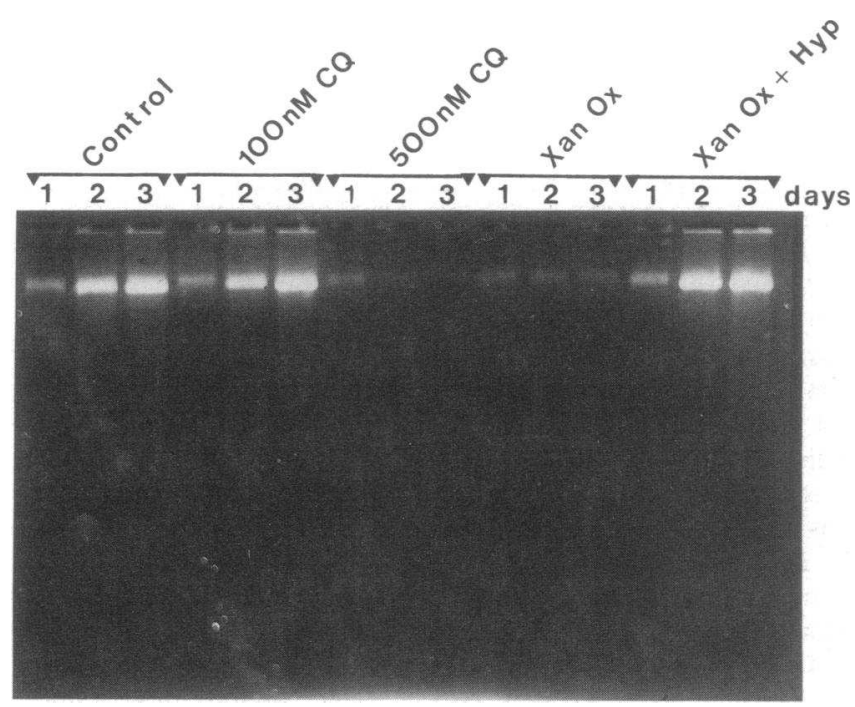

Figure 4. Confirmation by agarose gel electrophoresis of DNA accumulation in infected RBC, inhibition by xanthine oxidase, and reversal by hypoxanthine. Extracts from infected RBC, incubated for 1, 2, and $3 \mathrm{~d}$ under the conditions described in Fig. 3, were electrophoresed on a $2 \%$ agarose gel, and visualized with ethidium bromide. Nucleic acid consisted predominantly of a single band of high molecular weight genomic DNA, migrating close to the origin.

are anucleate, synthesize neither nucleic acid or protein, and are metabolically rather inactive. Hence the biochemical effects of rapidly growing and dividing Plasmodia are readily apparent, even though only a small percentage of erythrocytes are, in fact, infected.

We propose that xanthine oxidase exerts its inhibitory effect by depriving parasites of purine, in the form of hypoxanthine, upon which they depend for nucleic acid biosynthesis. A number of alternative possibilities should be considered. First,

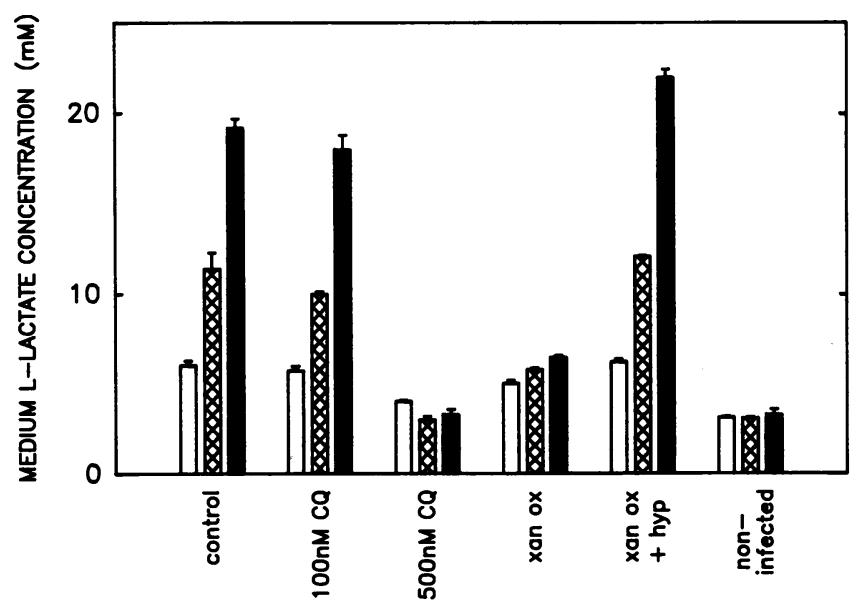

Figure 5. L-Lactate production by parasite-infected RBC, inhibition by xanthine oxidase and reversal by hypoxanthine. A deproteinized extract of spent medium, removed after the first ( $\square$ ), second $(\nabla)$, and third ( $\square$ ) 24-h incubation periods, in the experiment described in Fig. 3 was assayed for L-lactate. All assays were performed on duplicate samples. Error bars denote the difference between individual duplicates and their respective mean. 


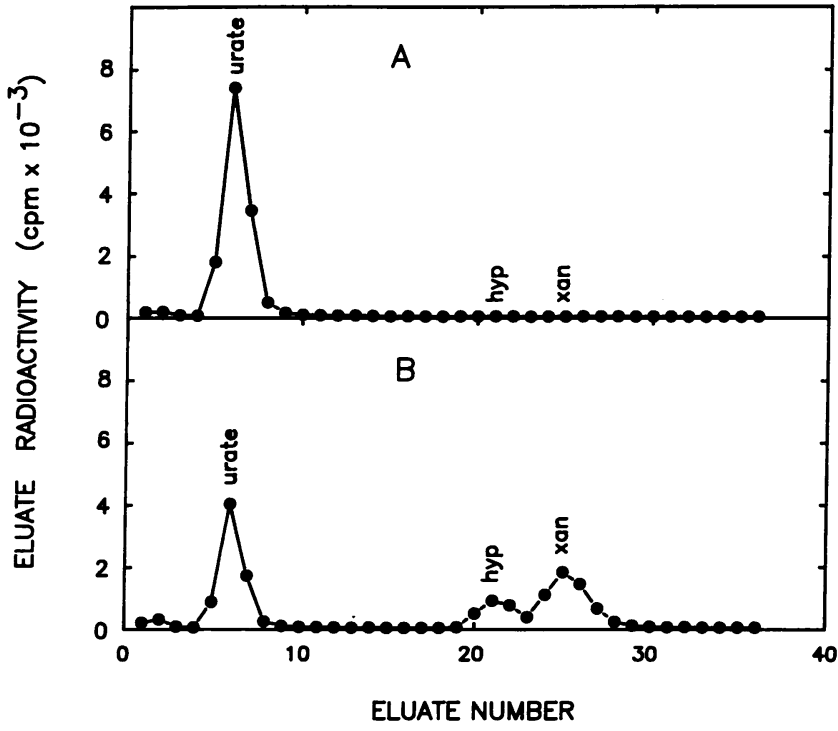

Figure 6. Extent of $1 \mathrm{mM}$ hypoxanthine oxidation after $24 \mathrm{~h}$ under hypoxanthine "rescue" conditions. Reverse-phase HPLC analyses of medium containing $3 \mathrm{mU} / \mathrm{ml}$ xanthine oxidase, incubated for $1 \mathrm{~h}$ with $25 \mu \mathrm{M}\left[{ }^{14} \mathrm{C}\right]$ hypoxanthine $(A)$, showed quantitative conversion of label to urate, whereas after $24 \mathrm{~h}$ of incubation with $1 \mathrm{mM}\left[{ }^{14} \mathrm{C}\right]-$ hypoxanthine $(B)$, significant hypoxanthine and xanthine remained detectable. The elution position of markers are superimposed on the radioactivity profile.

a toxic contaminant of the xanthine oxidase preparation used may have mediated growth inhibition. However, when the enzyme is exposed to $100^{\circ} \mathrm{C}$, loss of catalytic activity parallels loss of inhibitory activity, and confirms that a catalytically intact enzyme is required to produce inhibition. A further possibility is that active oxygen species, including superoxide anions and

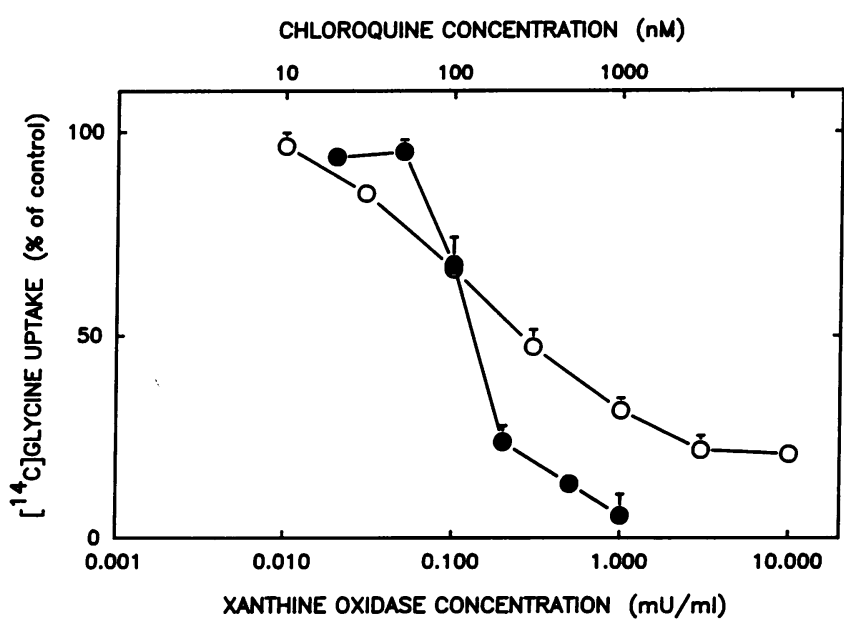

Figure 7. Dose-response curves for chloroquine and xanthine oxidase as inhibitors of parasite growth. Infected erythrocytes, at a $1 \%$ initial parasitemia, were incubated for $48 \mathrm{~h}$ at varying concentrations of chloroquine (๑), and xanthine oxidase ( 0 ). Parasite viability was assessed by $\left[{ }^{14} \mathrm{C}\right]$ glycine uptake, as described in Methods, and expressed as a percentage of the uptake observed in the absence of either inhibitor. Error bars denote the difference between duplicates and their mean. hydrogen peroxide, generated in the xanthine oxidase reaction, may mediate the toxic effect of the enzyme (30). Direct evidence to the contrary is provided by the inability of superoxide dismutase and catalase to relieve growth inhibition. Furthermore, inclusion of hypoxanthine with xanthine oxidase reversed, rather than aggravated, growth inhibition seen with xanthine oxidase alone. Generation of oxygen radicals would, if anything, be enhanced by provision of additional substrate for the xanthine oxidase reaction.

Most compelling evidence for hypoxanthine deprivation, however, comes from the ability of hypoxanthine to completely reverse inhibition of growth induced by xanthine oxidase. By adjusting the relative concentration of hypoxanthine and xanthine oxidase, we could achieve conditions where some hypoxanthine remained available for parasite use for the entire 24-h incubation period with xanthine oxidase, and showed that this was, in itself, sufficient to restore unimpeded parasite growth.

During the first $36 \mathrm{~h}$ of its 48 -h growth cycle, $P$. falciparum exists in uninucleate ring form, which thus predominates in unsynchronized cultures (10). During the final $12 \mathrm{~h}$ of the cycle, rings mature to trophozoites, which undergo cell division to form schizonts containing 8-32 nuclei (4). Each is capable of infecting a fresh erythrocyte, and initiating a new cycle. The accumulation of abnormal trophozoites and early schizonts induced by xanthine oxidase is consistent with failure of cell division due to lack of nucleic acid precursor. These morphological changes are reversed by provision of sufficient hypoxanthine to overcome the deficiency. Growth arrest at the same developmental stage is produced by $\alpha$-difluoromethylornithine, an inhibitor of polyamine synthesis, which prevents DNA replication (31). In this case, normal maturation of schizonts is restored by putrescine rescue (32).

The total purine nucleotide concentration and mean cell volume of human erythrocytes is approximately $1.8 \times 10^{-3} \mathrm{M}$ and $9.0 \times 10^{-14}$ liters, respectively $(33,34)$. This corresponds to $1.8 \times 10^{-3} \times 9.0 \times 10^{-14} \times 6.0 \times 10^{23}=1.0 \times 10^{8}$ purine molecules per erythrocyte. The size of the haploid genome of $P$. falciparum has been estimated at $3 \times 10^{8}$ base pairs (35). Since each base pair contains one purine, during a single cell division only a third of the purine complement of DNA can be supplied by the host erythrocyte. Considering that a replicating schizont typically produces not one, but 8-32 daughter nuclei, and that purine is also required for RNA synthesis, it becomes apparent that the infected cell can provide no more than $1-2 \%$ of its parasite's purine requirement. The remainder must be supplied from outside, and in our in vitro system at least, from adjacent noninfected red cells. Purine is released from red cells almost exclusively in the form of hypoxanthine $(23,24)$, and is thus susceptible to oxidation by xanthine oxidase present in the medium.

Failure of $1 \times 10^{-7} \mathrm{M}$ chloroquine to abolish parasite growth is indicative of chloroquine resistance (31). Thus, we have demonstrated that a chloroquine-resistant strain of $P$. falciparum is amenable to inhibition by xanthine oxidase. This has important implications. While new antimalarial drugs may be ineffective $a b$ initio against parasites bearing the multiple drug resistance (mdr) phenotype, which enhances efflux rate of a broad spectrum of antimalarial drugs approximately 50 -fold $(3,36)$, only the development of an entire de novo purine bio- 
synthetic pathway could render parasites resistant to hypoxanthine depletion.

These in vitro results must now be extended to the in vivo situation. The therapeutic potential of xanthine oxidase relies on the fact that, while the malaria parasite appears to depend entirely on hypoxanthine salvage, the human host is able to synthesize its purine requirements de novo. Potential problems associated with short term administration of xanthine oxidase include development of antibodies, tissue damage caused by oxygen radicals, and excessive production of uric acid. These adverse reactions may be minimised by the coadministration of superoxide dismutase (37), catalase (38), and/or uricase (39), which have been shown to be nontoxic when injected into animals and humans. Xanthine oxidase is abundant in milk, from which it may be purified relatively inexpensively. Preliminary experiments involving intravenous administration of xanthine oxidase to rabbits (unpublished observations) reveal an absence of untoward effects, apart from a transitory increase in plasma urate. If this proves true for humans, the more expensive uricase and oxygen radical scavenging enzymes may prove unnecessary, making xanthine oxidase a realistic option for malaria therapy in the Third World. Modification of xanthine oxidase by attachment of polyethylene glycol (38), or encapsulation in red cell ghosts (40), would shield it from immune detection and extend the circulating life, while retaining catalytic activity. Provided such formulations proved safe in experimental animals, their effect in combination with chloroquine for treatment of acute malaria should be compared to the clinical response to chloroquine alone. Use of an untried agent as sole therapy can not be justified in so serious a condition.

Although the ability of exogenous hypoxanthine to enhance growth of malaria parasites is generally well appreciated, the potential for removing endogenous hypoxanthine, and thereby inhibiting parasite growth, has not been explored. Our study shows that xanthine oxidase inhibits growth of $P$. falciparum, as assessed microscopically and by a number of independent biochemical parameters. Inhibition is specifically mediated by hypoxanthine depletion. We confirm that hypoxanthine is an obligate intermediate in the utilization of host derived purine, and propose that strategies aimed at depriving malaria parasites of hypoxanthine with xanthine oxidase in vivo may find application in treatment of malaria infections resistant to conventional forms of chemotherapy. Of interest in this regard are the recently discovered potent inhibitors of purine nucleoside phosphorylase (J. Sircar, unpublished observations), in that hypoxanthine formation in humans depends exclusively on the activity of this enzyme.

\section{Acknowledgments}

We thank the South African Medical Research Council for financial support, Lucille Odes for expert secretarial assistance, and Mervyn Berman for his critical appraisal of the manuscript.

\section{References}

1. Wyler, D. J. 1983. Malaria-resurgence, resistance and research. N. Engl. J. Med. 308:875-878.

2. WHO Scientific Group. 1984. Drug resistance in P. falciparum. In Advances in Malaria Chemotherapy. WHO Tech. Rep. Ser. 711:22-29.

3. Krogstad, D. J., I. Y. Gluzman, D. E. Kyle, A. M. J. Oduola, S. K. Martin, W. K. Milhous, and P. H. Schlesinger. 1987. Efflux of chloroquine from Plasmo- dium falciparum: mechanism of chloroquine resistance. Science (Wash. DC) 238:1283-1285

4. Markell, E. K., M. Voge, and D. T. John. 1986. Malaria. In Medical Parasitology. B. Venable, editor. W. B. Saunders Co., Philadelphia. 79-102.

5. Cox, F. E. G. 1991. Variation and vaccination. Nature (Lond.). 349:193.

6. WHO Scientific Group. 1987. Nucleic acid metabolism. In The Biology of Malaria Parasites. WHO Tech. Rep. Ser. 743:125-127.

7. Hassan, H. F., and G. H. Coombs. 1988. Purine and pyrimidine metabolism in parasitic protozoa. FEMS (Fed. Eur. Microbiol. Soc.) Microbiol. Rev. 54:47-84.

8. Scheibel, L. W., and I. W. Sherman. 1988. Purine transport and the salvage pathway. In Malaria Principles and Practice of Malariology. Volume I. W. H. Wernsdorfer and I. McGregor, editors. Churchill Livingstone, New York. 237239.

9. Webster, H. K., and Whaun, J. M. 1981. Purine metabolism during continuous erythrocyte culture of human malaria parasites (P. falciparum). Prog. Clin. Biol. Res. 55:557-573.

10. Trager, W., and J. B. Jensen. 1976. Human malaria parasites in continuous culture. Science (Wash. DC). 193:673-675.

11. Freese, J. A., B. L. Sharp, F. C. Ridl, and M. B. Markus. 1988. In vitro cultivation of Southern African strains of Plasmodium falciparum and gametocytogenesis. S. Afr. Med. J. 73:720-722.

12. Zolg, J. W., A. J. MacLeod, I. H. Dickson, and J. G. Scaife. 1982. Plasmodium falciparum: modification of the in vitro culture conditions improving parasitic yields. J. Parasitol. 68:1072-1080.

13. Divo, A. A., and J. B. Jensen. 1982. Studies on serum requirements for cultivation of Plamodium falciparum. 2. Medium enrichment. Bull. WHO. 60:571-575.

14. Bungener, W. 1974. Einfluss von allopurinol auf zyklusdauer und vermehrungsrate von Plasmodium vinckei in der ratte. Tropenmed. Parasit. 25:464468

15. Webster, H. K., W. P. Wiesmann, M. D. Walker, T. Bean, and J. M. Whaun. 1984. Hypoxanthine metabolism by human malaria infected erythrocytes: focus for the design of new antimalaria drugs. Adv. Exp. Med. Biol. 165A:219-223.

16. Chulay, J. D., J. D. Haynes, and C. L. Diggs. 1983. Plasmodium falciparum: assessment of in vitro growth by $\left[{ }^{3} \mathrm{H}\right]$ hypoxanthine incorporation. Exp. Parasitol. 55:138-146.

17. Daddona, P. E., W. P. Wiesmann, C. Lambros, W. N. Kelley, and H. K. Webster. 1984. Human malaria parasite adenosine deaminase. J. Biol. Chem. 259:1472-1475.

18. Daddona, P. E., W. P. Wiesmann, W. Milhous, J-W. Chern, L. B. Townsend, M. S. Hershfield, and H. K. Webster. 1986. Expression of human malaria parasite purine nucleoside phosphorylase in host enzyme-deficient erythrocyte culture. J. Biol. Chem. 261:11667-11673.

19. Webster, H. K., W. P. Wiesmann, and C. S. Pavia. 1984. Adenosine deaminase in malaria infection: effect of 2 '-deoxycoformycin in vivo. $A d v$. Exp. Med. Biol. 165A:225-229.

20. Al-Khalidi, U. A. S., and T. H. Chaglassian. 1965. The species distribution of xanthine oxidase. Biochem. J. 97:318-320.

21. Roth, E., N. Ogasawara, and S. Schulman. 1989. The deamination of adenosine and AMP in Plasmodium falciparum-infected human erythrocytes: in vitro use of 2 ' deoxycoformycin and AMP deaminase-deficient red cells. Blood. 74:1121-1125.

22. Berman, P., and L. Human. 1990. Regulation of 5-phosphoribosyl 1-pyrophosphate and of hypoxanthine uptake and release in human erythrocytes by oxypurine cycling. J. Biol. Chem. 265:6562-6568.

23. Bontemps, F., G. Van den Berghe, and H. G. Hers. 1986. Pathways of adenine nucleotide catabolism in erythrocytes. J. Clin. Invest. 77:824-830.

24. Berman, P. A., D. A. Black, L. Human, and E. H. Harley. 1988. Oxypurine cycle in human erythrocytes regulated by $\mathrm{pH}$, inorganic phosphate and oxygen. J. Clin. Invest. 82:980-986.

25. Henderson, J. F., L. W. Brox, W. N. Kelley, F. M. Rosebloom, and J. E Seegmiller. 1968. Kinetic studies of hypoxanthine-guanine phosphoribosyltransferase. J. Biol. Chem. 243:2514-2522.

26. Roth, E. F., R. M. Ruprecht, S. Schulman, J. Vanderberg, and J. A. Olsen. 1986. Ribose metabolism and nucleic acid synthesis in normal and glucose-6-phosphate dehydrogenase-deficient human erythrocytes infected with Plasmodium falciparum. J. Clin. Invest. 77:1129-1135.

27. Yayon, A., Z. I. Cabantchik, and H. Ginsburg. 1984. Identification of the acidic compartment of Plasmodium falciparum-infected human erythrocytes as the target of the antimalarial drug chloroquine. EMBO (Eur. Mol. Biol. Organ.). J. 3:2695-2700.

28. Maniatis, T., E. F. Fritsch, and J. Sambrook. 1982. Appendix A: Biochemical techniques. In Molecular Cloning-a Laboratory Manual. Cold Spring Harbor Laboratory, Cold Spring Harbor, NY. 468.

29. Bergmeyer, H. U. 1974. L-lactate. In Methods in Enzymatic Analysis. 2nd English edition. Vol. III. Academic Press, Inc., New York. 1464-1468. 
30. McCord, J. M. 1985. Oxygen-derived free radicals in postischaemic tissue injury. N. Engl. J. Med. 312:159-163.

31. Bitonti, A. J., J. A. Dumont, T. L. Bush, M. L. Edwards, D. M. Stemerick P. P. McCann, and A. Sjoerdsma. 1989. Bis(benzyl)polyamine analogs inhibit the growth of chloroquine-resistant human malaria parasites in vitro and in combination with $\alpha$-difluoromethylornithine can cure murine malaria. Proc. Natl. Acad. Sci. USA. 86:651-655.

32. Assaraf, Y. G., J. Golenser, D. T. Spira, and U. Bachrach. 1986. Plasmodium falciparum: synchronization of culture with DL- $\alpha$-difluoromethylornithine, an inhibitor of polyamine biosynthesis. Exp. Parasitol. 61:229-235.

33. Simmonds, H. A., L. D. Fairbanks, G. S. Morris, D. R. Webster, and E. H Harley. 1988. Altered erythrocyte nucleotide patterns are characteristic of inherited disorders of purine and pyrimidine metabolism. Clin. Chim. Acta. 171:197-210.

34. Dacie, J. V., and S. M. Lewis. 1984. Haematological values in health. In Practical Haematology. 6th edition. Churchill Livingstone, New York. 8.
35. Hough-Evans, B. R., and J. Howard. 1982. Genome size and DNA complexity of Plasmodium falciparum. Biochim. Biophys. Acta. 698:56-61.

36. Newbold, C. 1990. The path of drug resistance. Nature (Lond.). 345:202203.

37. Pyatak, P. S., A. Abuchowski, and F. F. Davis. 1980. Preparation of a polyethylene glycol:superoxide dismutase adduct, and an examination of its blood circulating life and anti-inflammatory activity. Res. Commun. Chem. Pathol. Pharmacol. 29:113-127.

38. Abuchowski, A., J. R. McCoy, N. C. Palczuk, T. van Es, and F. F. Davis. 1976. Effect of covalent attachment of polyethylene glycol on immunogenicity and circulating life of bovine liver catalase. J. Biol. Chem. 252:3582-3586.

39. Chen, R. H.-L., A. Abuchowski, T. van Es, N. C. Palczuk, and F. F. Davis. 1981. Properties of 2 urate oxidases modified by the covalent attachment of polyethylene glycol. Biochim. Biophys. Acta. 660:293-298.

40. Adriaenssens, K., D. Karcher, A. Lowenthal, and H. G. Terheggen. 1976. Use of enzyme loaded erythrocytes in in vitro correction of arginine-deficient erythrocytes in familial hyperargininaemia. Clin. Chem. 22:323-326. 